\title{
MicroRNA-524-5p regulates the proliferation and invasion of HTR-8/SVneo trophoblasts by targeting NUMB in the Notch signaling pathway
}

\author{
LINMEI ZHENG ${ }^{*}$, JIE SONG* ${ }^{*}$ RONG TANG, XIAOJU CHEN, LI WANG, DONGCAI WU, HUI CEN and LEI SHI \\ Department of Obstetrics, Hainan General Hospital, Hainan Affiliated Hospital of \\ Hainan Medical University, Haikou, Hainan 570311, P.R. China
}

Received November 7, 2019; Accepted October 19, 2020

DOI: $10.3892 / \mathrm{mmr} .2021 .12075$

\begin{abstract}
Preeclampsia is a pregnancy disorder that is primarily associated with maternal and neonatal or fetal morbidity and mortality. The discovery of dysregulated microRNAs (miRs) and their roles in preeclampsia has provided new insight into the mechanisms involved in pregnancy-related disorders. In the present study, quantitative PCR demonstrated that the expression levels of miR-524-5p were lower in patients with preeclampsia than those in normal pregnant women. Cell Counting Kit- 8 and Transwell assays indicated that overexpression of miR-524-5p promoted the proliferation and invasion of HTR-8/SVneo cells, whereas inhibition of miR-524-5p suppressed HTR-8/SVneo cell proliferation and invasion. Furthermore, NUMB endocytic adaptor protein (NUMB), a negative regulator of the Notch signaling pathway and a target gene of miR-524-5p, limited the effects of miR-524-5p on HTR-8/SVneo cell invasion and migration. The present study demonstrated that $\mathrm{miR}-524-5 \mathrm{p}$ regulated the proliferation and invasion of HTR-8/SVneo cells at least partly by targeting NUMB to regulate the Notch signaling pathway.
\end{abstract}

\section{Introduction}

Preeclampsia is a common serious disorder of obstetrics and is a hypertensive complication that occurs after the 20th week of gestation, characterized by new-onset hypertension (1).

Correspondence to: Miss Lei Shi, Department of Obstetrics, Hainan General Hospital, Hainan Affiliated Hospital of Hainan Medical University, 19 Longhua Road, Haikou, Hainan 570311, P.R. China

E-mail: poipoi1010@163.com

*Contributed equally

Abbreviations: NUMB, NUMB endocytic adaptor protein; PCNA, proliferating cell nuclear antigen

Key words: preeclampsia, proliferation, invasion, microRNA, NUMB, Notch
The primary cause of maternal mortality in preeclampsia has not yet been elucidated. At present, it is hypothesized that placental maternal surface spiral arterial remodeling disorder and insufficient extravillous trophoblast invasion are key factors leading to preeclampsia $(2,3)$.

MicroRNAs (miRNAs/miRs) are a type of small non-coding RNAs that have been reported to be post-transcriptional gene regulators and tumor suppressors $(4,5)$. Previous studies have investigated the role of miR-524-5p in numerous types of cancer. For example, Chen et al (6) demonstrated that high/low miR-524-5p expression is associated with improved/worse survival rate and pathological grade of patients with glioma, and Liu et al (7) detected lower levels of miR-524-5p expression in human melanoma, whereas overexpression of miR-524-5p effectively inhibited melanoma cell proliferation and migration. Furthermore, Liu et al (7) demonstrated that tumors overexpressing miR-524-5p were significantly smaller than those in negative control (NC) mice. Additionally, a previous study has demonstrated that miR-524-5p expression is downregulated in preeclampsia (8). However, the role of miR-524-5p in preeclampsia has not been fully elucidated.

NUMB endocytic adaptor protein (NUMB), a cell fate determinant, serves an important role in asymmetric cell division (9). NUMB is a key negative regulator of the Notch signaling pathway (10) and is involved in numerous physiological processes, such as differentiation/proliferation balance, apoptosis regulation, cell migration and tissue regeneration (11-13). To the best of our knowledge, NUMB expression in placental tissues with extremely active growth and differentiation has not yet been identified. Therefore, the present study investigated the potential roles of miR-524-5p and NUMB in trophoblast proliferation and invasion.

\section{Materials and methods}

Patients. Patients at Hainan Provincial People's Hospital (Haikou, China) were enrolled in the present study between September 2017 and January 2019. A total of 40 patients with preeclampsia and 40 healthy pregnant women were admitted in the present study. The risk factors for the development of pre-eclampsia were recorded according to a previous study (14). According to the American College of Obstetricians 
and Gynecologists practice bulletin for diagnosis and management of preeclampsia and eclampsia, severe preeclampsia was defined as either sustained systolic blood pressure $\geq 160 \mathrm{mmHg}$ and/or diastolic blood pressure $\geq 110 \mathrm{mmHg}$ (measured twice, $\geq 6 \mathrm{~h}$ apart) or severe proteinuria ( $>5 \mathrm{~g} / 24 \mathrm{~h}$ specimen or $\geq 3 \mathrm{~g} / \mathrm{l}$ in $\geq 2$ random samples collected $4 \mathrm{~h}$ apart) (15). Normal systolic blood pressure is $<140 \mathrm{mmHg}$ and diastolic blood pressure is $<110 \mathrm{mmHg}$. Age, BMI and smoking history were also recorded but were not used as exclusion criteria. Patients with non-severe preeclampsia according to the criterion listed in the International Society for the Study of Hypertension in Pregnancy were excluded. Clinical information was recorded up to the expected date of delivery and the placenta was obtained for follow-up study. The present study was approved by the Ethics Committee of Hainan General Hospital and all patients provided written informed consent.

Cell culture. The human HTR-8/SVneo trophoblast cell line was purchased from Shanghai Enzyme Research Biotechnology Co., Ltd. HTR-8/SVneo cells were seeded in a 10-cm cell culture dish with DMEM medium (Gibco; Thermo Fisher Scientific, Inc.) containing 10\% FBS (Gibco; Thermo Fisher Scientific, Inc.) and $1 \%$ penicillin/streptomycin at $37^{\circ} \mathrm{C}$ in a humidified atmosphere with $5 \% \mathrm{CO}_{2}$.

miR inhibitor and mimic transfection. miR-524-5p inhibitor, mimic and negative control (NC) oligonucleotides $(20 \mu \mathrm{M}$; Guangzhou RiboBio Co., Ltd.) were transfected into cells using $5 \mu 1$ Lipofectamine ${ }^{\circledR} 2000$ (Invitrogen; Thermo Fisher Scientific, Inc.) in 6-well plates at $37^{\circ} \mathrm{C}$ for $24 \mathrm{~h}$. The cells were divided into four groups: Scrambled Inhibitor NC (5'-GAGAAAGUG CUUCGGUUUUUUG-3'), miR-524-5p inhibitor (5'-GAG AAAGUGCUUCCCUUUGUAG-3'), scrambled mimic NC (5'-CAAAAAACCGAAGCACUUUCUC-3') and miR-524-5p mimic (5'-CUACAAAGGGAAGCACUUUCUC-3').

Cell counting Kit-8 (CCK-8) assay. Cells were seeded into 6 -well plates at a density of $1 \times 10^{4}$ cells $/ \mathrm{ml}$ for $0,24,48$ or $72 \mathrm{~h}$. Cells were incubated with $10 \mu \mathrm{lCCK}-8$ solution (Beijing Solarbio Science \& Technology Co., Ltd.) at $37^{\circ} \mathrm{C}$ for $2 \mathrm{~h}$, and cell viability was measured. Absorbance values were measured using a microplate reader at a wavelength of $450 \mathrm{~nm}$.

Bioinformatics analysis. The target genes of miR-524-5p were predicted using TargetScan (http://www.targetscan. org/mamm_31/) and microRNA.org databases (http://www. mirbase.org/) (16,17). Binding sites between miR-524-5p and the target genes were searched to identify potential interactions between them.

Luciferase reporter assay. A luciferase reporter assay was conducted to explore the association between miR-524-5p and NUMB, as described previously (18). HTR-/SVneo cells were divided into two groups, wild-type and mutant NUMB with 3'-untranslated region (UTR) reporters. HTR-8/SVneo cells at $80 \%$ confluence were co-transfected at $37^{\circ} \mathrm{C}$ for $24 \mathrm{~h}$ with wild-type or mutant NUMB 3'-UTR reporters $(16 \mu \mathrm{g} / \mathrm{ml}$; Generay Biotech Co., Ltd.) together with miR-524-5p mimic $\left(50 \mathrm{pmol} / \mathrm{ml}\right.$ ) or NC using Lipofectamine ${ }^{\circledR} 2000$ (Invitrogen; Thermo Fisher Scientific, Inc.). Subsequently, Dual-Luciferase
Reporter Assay System (Promega Corporation) was performed according to the manufacturer's protocol. Cells were harvested and lysed for the assay $24 \mathrm{~h}$ after transfection. Renilla luciferase was used to normalize the data.

Transwell invasion assay. Transwell assays were carried out as described previously (19). Cells $\left(2 \times 10^{4}\right.$ cells/well) were cultured at $37^{\circ} \mathrm{C}$ for $24 \mathrm{~h}$ in 24 -well plates with Transwell inserts precoated with Matrigel ${ }^{\circledR}\left(37^{\circ} \mathrm{C}\right.$ for $30 \mathrm{~min}$; BD Pharmingen; BD Biosciences). DMEM medium was added to the upper chamber, and DMEM containing 10\% FBS in the lower chamber. Subsequently, cells were stained with $0.1 \%$ crystal violet (Sigma-Aldrich; Merck KGaA) at $20^{\circ} \mathrm{C}$ for $10 \mathrm{~min}$ and the number of cells that had invaded through the Matrigel membrane was counted using a light microscope (magnification, x100; Leica Microsystems GmbH).

Reverse transcription-quantitative $(R T-q) P C R$ analysis. Total RNA was extracted from cells using TRIzol ${ }^{\circledR}$ reagent and quantified using NanoDrop 2000c (both Thermo Fisher Scientific, Inc). For miR-524-5p detection, RNA was reverse transcribed to cDNA using the RevertAid First Strand cDNA kit (Thermo Fisher Scientific, Inc), according to the manufacturer's protocol. qPCR was performed in 96-well plates in the ABI Step-One plus Real-Time PCR system (Thermo Fisher Scientific, Inc.) using SYBR Green Master Mix (Takara Biotechnology Co.,Ltd.). Expression levels of miR-524-5p were normalized to those of small nuclear RNA U6, while GAPDH was used as an endogenous control for NUMB. Primers were obtained from Invitrogen (Thermo Fisher Scientific, Inc.). The primer sequences were as follows: miR-524-5p forward, 5'-CTACAAAGGGAAGCACTTTTCTCAA-3' and reverse, 5'-GTGCAGGGTCCGAGGT-3'; U6 forward, 5'-CGCTTC GGCAGCACATATAC-3' and reverse, 5'-CAGGGGCCATGC TAATCTT-3'; NUMB forward, 5'-AAGGCTTCTTTGGAA AAACTGG-3' and reverse, 5'-CATGGCTCAACCTTTCAC CT-3'; and GAPDH forward, 5'-TGTTCGTCATGGGTGTGA AC-3' and reverse, 5'-ATGGCATGGACTGTGGTCAT-3'. Each well contained $1 \mu \mathrm{l}$ template, $10 \mu \mathrm{l}$ Master Mix, $0.5 \mu \mathrm{l}$ forward primer $(10 \mu \mathrm{M}), 0.5 \mu \mathrm{l}$ reverse primer $(10 \mu \mathrm{M})$ and $8 \mu \mathrm{l}$ diethyl pyrocarbonate $\mathrm{H}_{2} \mathrm{O}$ in a $20-\mu 1$ reaction system. qPCR thermocycling conditions were as follows: Initial denaturation at $95^{\circ} \mathrm{C}$ for $2 \mathrm{~min}$, followed by 40 cycles of denaturation at $95^{\circ} \mathrm{C}$ for $20 \mathrm{sec}$, annealing at $60^{\circ} \mathrm{C}$ for $45 \mathrm{sec}$ and extension at $72^{\circ} \mathrm{C}$ for $30 \mathrm{sec}$. Finally, gene expression levels were calculated using the $2^{-\Delta \Delta \mathrm{Cq}}$ method (20).

Western blotting. Proteins were extracted from cells using RIPA buffer (EMD Millipore) and quantified using a BCA kit (Beijing Solarbio Science \& Technology Co., Ltd.). Target proteins $(30 \mu \mathrm{g})$ were separated according to their mass via $10 \%$ SDS-PAGE, then transferred to a PVDF membrane. Membranes were incubated with primary antibodies against proliferating cell nuclear antigen (PCNA; 1:1,000; cat. no. ab92552), Ki67 (1:2,000; cat. no. ab92742), NUMB (1:1,000; cat. no. ab220362), Bcl-2 (1:500; cat. no. ab196495), Notch1 (1:1,000; cat. no. ab52627), cyclin D1 (1:500; cat. no. ab40754), CDK6 (1:2,000; cat. no. ab151247) and GAPDH (1:10,000; cat. no. ab181602) (all Abcam) at $4^{\circ} \mathrm{C}$ overnight, then incubated with horseradish peroxidase-conjugated goat 
Table I. Clinical information of patients with PE and normal pregnant females.

\begin{tabular}{lccc}
\hline Parameter & Control $(\mathrm{n}=40)$ & PE $(\mathrm{n}=40)$ & P-value \\
\hline Maternal age at delivery, years & $29.53 \pm 2.90$ & $30.43 \pm 3.15$ & 0.1878 \\
Gestational age, weeks & $38.18 \pm 2.24$ & $36.70 \pm 3.15$ & $<0.05$ \\
Proteinuria, g/24 h & - & $4.57 \pm 1.40$ & $<0.001$ \\
Systolic blood pressure, mmHg & $105.81 \pm 13.41$ & $168.43 \pm 5.43$ & $<0.001$ \\
Diastolic blood pressure, mmHg & $77.38 \pm 4.33$ & $116.06 \pm 3.75$ & $<0.001$ \\
Fetal birth weight, g & $3,556.88 \pm 309.88$ & $2,689.85 \pm 428.90$ & $<0.001$ \\
BMI & $23.42 \pm 3.28$ & $23.01 \pm 3.66$ & 0.594 \\
Number of individuals who smoked, $n$ & 3.00 & 4.00
\end{tabular}

Data are expressed as the mean \pm standard deviation, unless otherwise stated. Significance was calculated between control and PE groups. PE, preeclampsia.

anti-rabbit IgG secondary antibody (1:5,000; cat. no. A0208; Beyotime Institute of Biotechnology) at $25^{\circ} \mathrm{C}$ for $1 \mathrm{~h}$. Bands were visualized using the ECL reagent (Merck KGaA) and density was measured via ImageJ software (v 2.1.4.7).

Statistical analysis. Data are presented as the mean \pm standard deviation of three independent experiemnts. Statistical comparisons were performed using SPSS 17.0 software (SPSS, Inc.) Differences between two groups were compared using an unpaired Student's t-test. One-way ANOVA followed by Tukey's multiple comparison test was used for comparisons among three groups. Pearson's correlation analysis was used to examine the correlation between NUMB and miR-524-5p expression. The $\chi^{2}$ test was used to analyze the number of volunteers who smoked. $\mathrm{P}<0.05$ was considered to indicate a statistically significant difference.

\section{Results}

miR-524-5p is downregulated in preeclamptic placenta compared with in normal placenta. A total of 40 patients with preeclampsia and 40 normal pregnant females (controls) were enrolled in the present study. Clinical characteristics of patients and normal pregnant females were recorded, including age, BMI, smoking history and systolic/diastolic blood pressure (Table I). Maternal age, BMI and smoking history exhibited no significant difference between the two groups. Patients with preeclampsia had a significantly shorter gestational age and lower fetal birth weight, while systolic and diastolic blood pressure in patients with preeclampsia were significantly higher than in normal controls. qPCR demonstrated that expression levels of miR-524-5p were significantly lower in patients with preeclampsia than in normal controls (Fig. 1), which indicated that downregulation of miR-524-5p expression may be a biomarker of preeclampsia.

miR-524-5p regulates proliferation and invasion of human HTR-8/SVneo trophoblasts. In order to investigate the effect of miR-524-5p on the proliferative and invasive abilities of human HTR-8/SVneo trophoblasts, miR-524-5p mimic and inhibitor were transfected into cells. Expression levels of miR-524-5p were significantly higher in the miR-524-5p

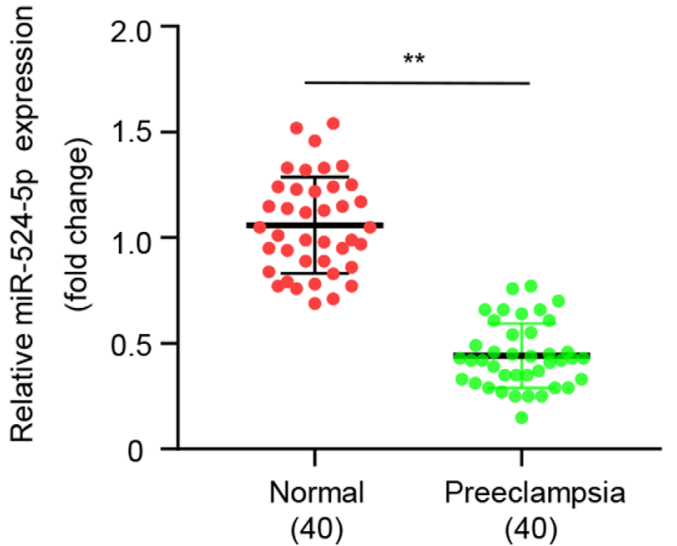

Figure 1. Relative miR-524-5p expression levels in controls and patients with preeclampsia. ${ }^{* *} \mathrm{P}<0.001$. miR, microRNA.

mimic group than in the mimic NC group (Fig. 2A) and significantly lower in the miR-524-5p inhibitor group than in the inhibitor NC group (Fig. 2B), which indicated that mimic and inhibitor had been transfected successfully. The CCK-8 assay demonstrated that cell viability of the miR-524-5p mimic group was significantly higher than that of the mimic NC group at $72 \mathrm{~h}$ (Fig. 2C), while cell viability of the miR-524-5p inhibitor group was significantly lower compared with the inhibitor NC group (Fig. 2D). Expression levels of PCNA and Ki67 were determined by western blotting to investigate the underlying mechanism of cell proliferation. Overexpression of miR-524-5p increased PCNA and Ki67 protein expression, while PCNA and Ki67 expression in the miR-524-5p inhibitor group was lower than in the inhibitor $\mathrm{NC}$ group, which demonstrated that inhibition of miR-524-5p inhibited cell proliferation (Fig. 2E).

In order to investigate the influence of miR-524-5p on the invasive ability of human HTR-8/SVneo trophoblasts, the degree of cell invasion was determined by Transwell invasion assay. Overexpression and inhibition of miR-524-5p in HTR-8/SVneo cells regulated cell invasion. After $24 \mathrm{~h}$, the invasive ability of cells in the miR-524-5p mimic group was significantly increased compared with that in the mimic $\mathrm{NC}$ group (Fig. 2F), while the invasive ability of cells in the 
A

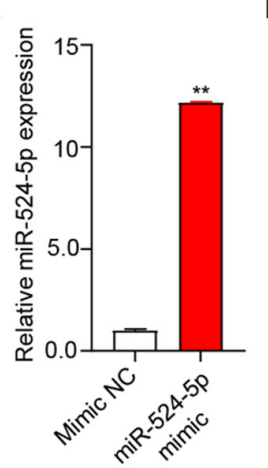

B

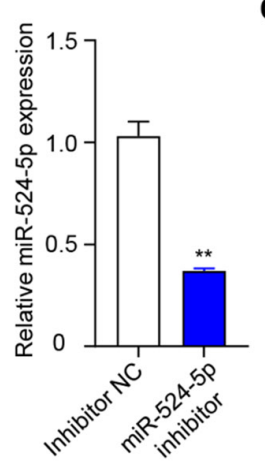

C

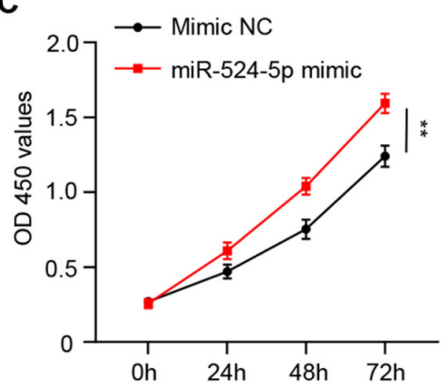

D

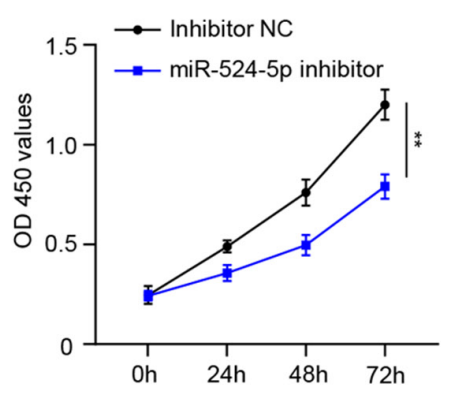

E

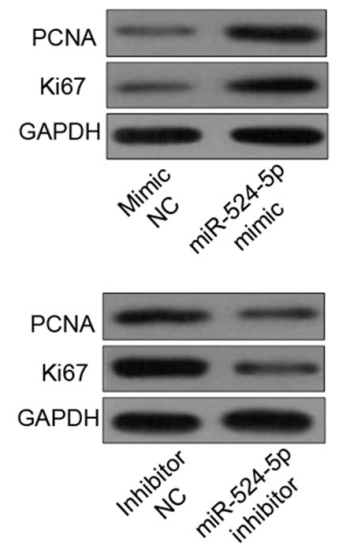

F

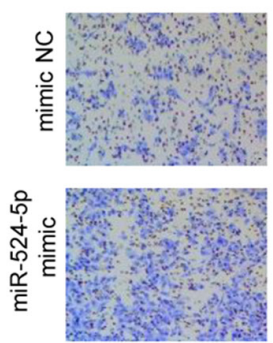

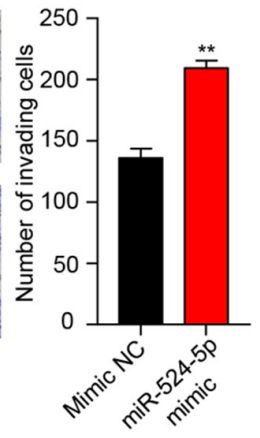

G

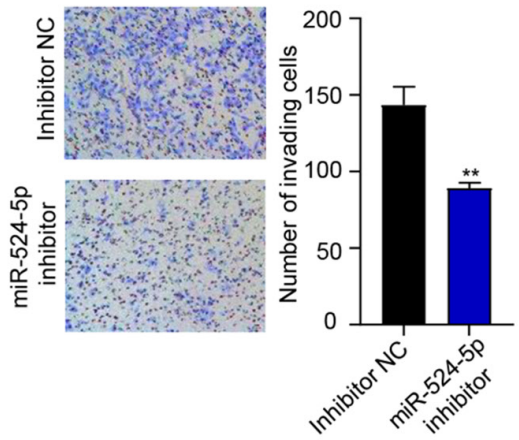

Figure 2. Low expression levels of miR-524-5p impede trophoblast cell proliferation and invasion. Relative miR-524-5p expression levels in HTR-8/SVneo cells following transfection with (A) miR-524-5p mimic and (B) inhibitor. Viability of HTR-8/SVneo cells transfected with (C) miR-524-5p mimic and (D) inhibitor for 0, 24, 48 and $72 \mathrm{~h}$. (E) Western blot analysis of PCNA and Ki67 protein levels in HTR-8/SVneo cells transfected with miR-145-5p mimic or NC, and miR-145-5p inhibitor or NC. Transwell invasion assay was performed to assess the invasiveness of HTR-8/SVneo cells transfected with (F) miR-145-5p mimic or NC or (G) miR-145-5p inhibitor or NC. Scale bar, $100 \mu \mathrm{m} .{ }^{* *} \mathrm{P}<0.01$; miR, microRNA; PCNA, proliferating cell nuclear antigen; NC, negative control; OD, optical density.

miR-524-5p inhibitor group was significantly decreased compared with that in the inhibitor NC group (Fig. 2G).

miR-524-5p regulates the expression levels of $N U M B$ via binding to the 3'-UTR of NUMB $m R N A$. In order to determine the target genes of miR-524-5p, TargetScan and microRNA. org databases were used. Results from the databases indicated that NUMB was a candidate target gene regulated by miR-524-5p, and microRNA.org database was used to predict the binding sites (Fig. 3A).

In order to determine whether inhibition of NUMB by miR-524-5p occurred via these predicted miR-524-5p binding sites, the binding site was mutated. The luciferase reporter assay indicated that transfection with the NUMB mutant 3'-UTR did not lead to a reduction in the relative luciferase activity in the presence of miR-524-5p, compared with miR NC (Fig. 3B). Subsequently, the expression levels of NUMB were determined following miR-524-5p mimic or inhibitor treatment. Inhibition of miR-524-5p increased the expression levels of NUMB mRNA and protein (Fig. 3C and E); conversely, when miR-524-5p was overexpressed, NUMB mRNA and protein expression levels were decreased (Fig. 3D and F). Additionally, Pearson's correlation analysis showed that NUMB exhibited a negative correlation with miR-524-5p (Fig. 3G), which suggested that the highly conserved sequence of the NUMB 3'-UTR may be the primary binding site of miR-524-5p, and further confirmed that miR-524-5p directly inhibited NUMB.

miR-524-5p regulates proliferation and invasion of HTR-8/SVneo cells by targeting NUMB to regulate the Notch signaling pathway. pcDNA3.1-NUMB and miR-524-5p mimic were transfected into human HTR-8/SVneo trophoblasts. Cells were divided into 4 groups, including mimic $\mathrm{NC}+$ pcDNA 3.1, miR-524-5p mimic + pcDNA 3.1, mimic $\mathrm{NC}+$ pcDNA 3.1-NUMB and miR-524-5p mimic + pcDNA 3.1-NUMB. Cell viability was determined by CCK-8 assay, and the results revealed that cell viability was highest in the miR-524-5p mimic + pcDNA 3.1 group and lowest in mimic $\mathrm{NC}+$ pcDNA 3.1-NUMB, which indicated that activation of miR-524-5p may inhibit the expression levels of NUMB and cell viability was increased due to elevated miR-524-5p expression at $72 \mathrm{~h}$ (Fig. 4A).

Apoptosis rate is significantly increased in the syncytiotrophoblast in preeclampsia (21). The effect of miR-524-5p and NUMB on cell proliferation was investigated. PCND and Ki67 are common proliferation markers in apoptosis (22), and therefore western blot analysis was performed to detect 
A

NUMB (1068-1074 of NUMB 3' UTR)

\begin{tabular}{|c|c|}
\hline NUMB-WT & $\begin{array}{l}\text { 5' AGGUUACCAUAUUGGUUUC } \\
\text { 3“ CUCUUUCACGA--AGGGAAA } \\
\text { 3' }\end{array}$ \\
\hline & AUAUCGACUAUAUAUAUUC \\
\hline
\end{tabular}

B

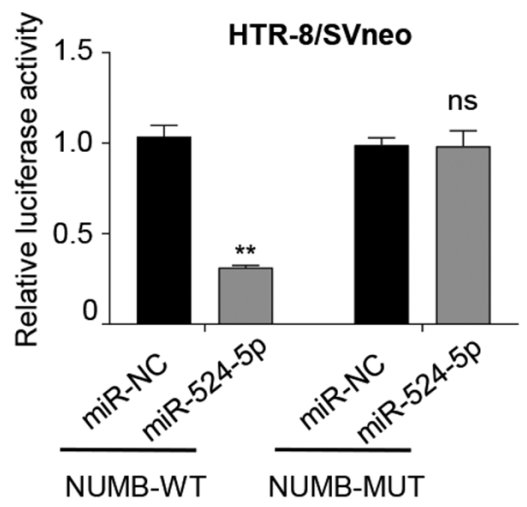

C

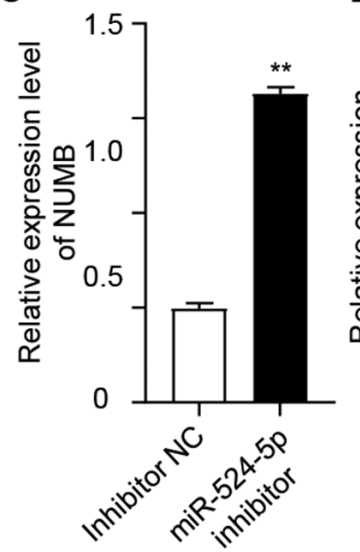

D

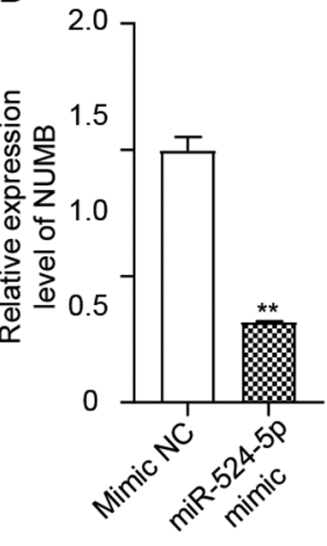

E

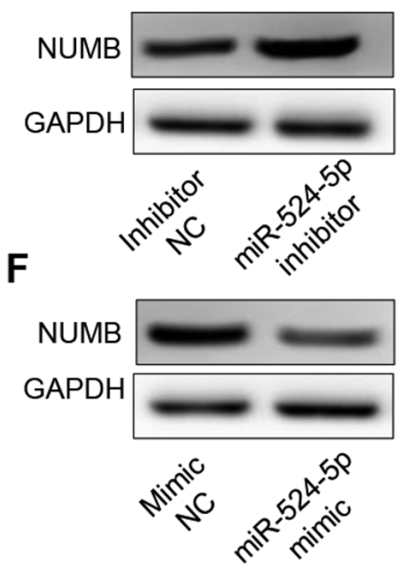

G

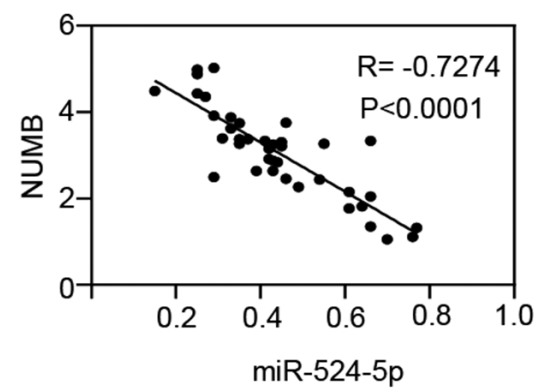

Figure 3. miR-524-5p regulates the expression levels of NUMB. (A) microRNA.org database was used to predict binding sites between miR-524-5p and NUMB. (B) Fluorescence intensity was measured using a dual luciferase assay. Overexpressed miR-524-5p bound to NUMB-WT and fluorescence intensity was weakened. NUMB-MUT exhibited no significant difference in fluorescence intensity. RT-qPCR was used to detect NUMB expression in the (C) inhibitor and (D) mimic groups. Compared with the inhibitor NC group, NUMB expression was significantly upregulated in the miR-524-5p inhibitor group, while compared with the mimic NC group, NUMB expression in the miR-524-5p mimic group was significantly downregulated. Western blotting was used to detect NUMB expression in the (E) inhibitor and (F) mimic groups. Compared with the inhibitor NC group, NUMB expression was significantly upregulated in the miR-524-5p inhibitor group, while it was significantly downregulated in the miR-524-5p mimic group compared with the mimic NC group. (G) RT-qPCR was used to detect the expression levels of NUMB in 40 preeclampsia tissues and Pearson's correlation coefficient was used to analyze the correlation between NUMB and miR-524-5p expression. There was a significant negative association between NUMB and miR-524-5p expression. ${ }^{* *} \mathrm{P}<0.01$. miR, microRNA; NUMB, NUMB endocytic adaptor protein; WT, wild-type; MUT, mutant; RT-q, reverse transcription-quantitative; NC, negative control; UTR, untranslated region; ns, not significant.

the expression levels of these two proteins. Expression levels of PCNA and Ki67 were highest in the miR-524-5p mimic + pcDNA 3.1 group and lowest in the mimic $\mathrm{NC}+$ pcDNA 3.1-NUMB group, which indicated that overexpression of miR-524-5p decreased NUMB and increased the cell proliferative ability (Fig. 4B). Additionally, the effect of miR-524-5p mimic on cell invasion was investigated. The results revealed that the miR-524-5p mimic + pcDNA3.1 group had the highest number of invading cells and the mimic NC + pcDNA3.1-NUMB group exhibited the lowest number. The number of invading cells in the miR-524-5p mimic + pcDNA3.1-NUMB was between the two aforementioned groups (Fig. 4C and D).

The present results suggested that miR-524-5p mimic inhibited NUMB expression and that high expression levels of NUMB inhibited cell proliferation and invasion. As the mechanism remains unknown, the present study investigated the specific mechanism by which miR-524-5p stimulates proliferation and invasion of HTR-8/SVneo cells by detecting the expression levels of signaling molecules associated with the Notch signaling pathway. Protein expression levels of Bcl-2, Notch, cyclin D1 and CDK6 were determined by western blotting. Protein expression level trends of Bcl-2, Notch, cyclin D1 and CDK6 in each group were downregulated when pcDNA 3.1-NUMB were transformed simultaneously with NC or mimic (Fig. 4E and S1). This indicated that miR-524-5p stimulated proliferation and invasion of HTR-8/SVneo cells by targeting NUMB to regulate the Notch signaling pathway.

\section{Discussion}

In recent years, numerous studies have continued to investigate preeclampsia $(1,23)$, but the exact cause has not yet been elucidated. There is still no ideal animal model that replicates all pathological conditions of preeclampsia that are produced. Preeclampsia is hypothesized to be associated with insufficient cell invasion and endothelial cell dysfunction (24). In the present study, patients with severe PE symptoms exhibited higher systolic and diastolic blood 
A

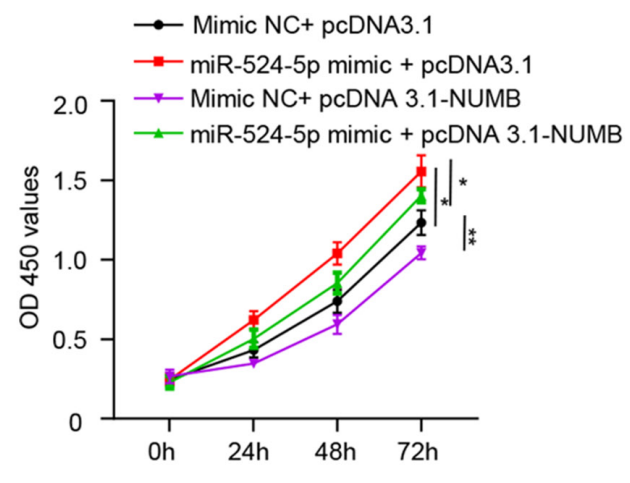

B

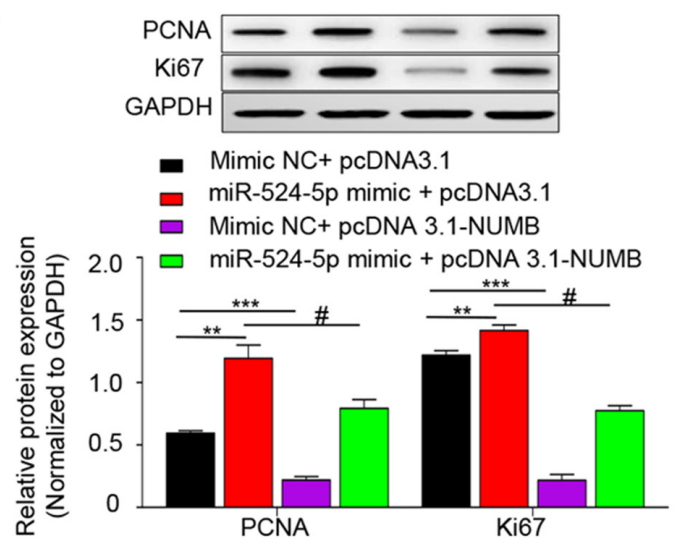

D

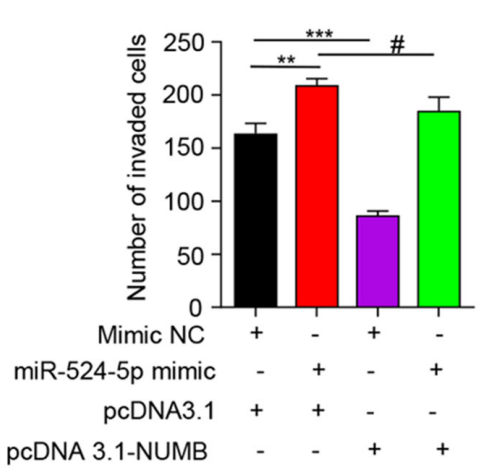

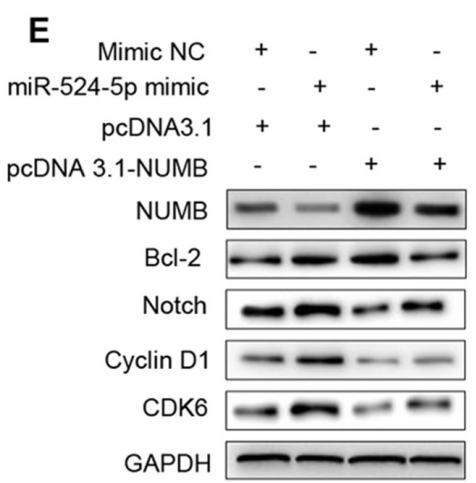

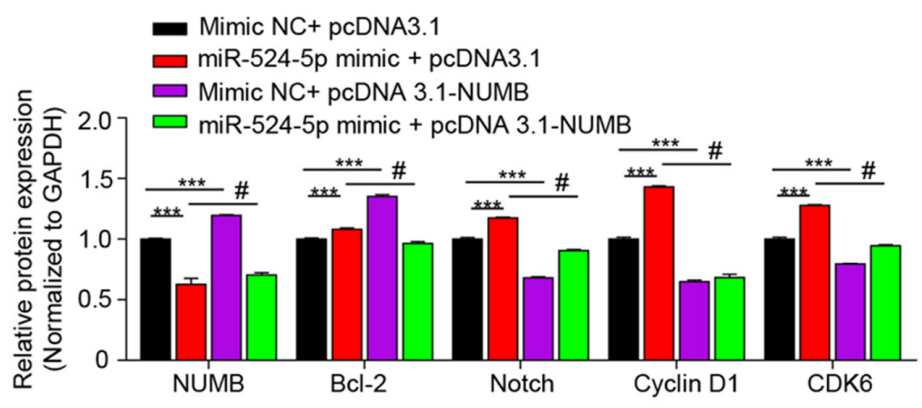

Figure 4. miR-524-5p regulates the Notch signaling pathway via NUMB. (A) Cell Counting Kit-8 was used to detect the proliferation ability of each group for 0, 24, 48 and $72 \mathrm{~h}$. (B) Western blot analysis of PCNA and Ki67 protein expression. (C) Invasive ability was detected using Transwell assay (Scale bar, $100 \mu \mathrm{m}$ ) and (D) quantified. (E) Western blot was used to detect the expression levels of NUMB, Notch1, Bcl-2, Cyclin D1 and CDK6 in the Notch signaling pathway. ${ }^{*} \mathrm{P}<0.05 ;{ }^{* *} \mathrm{P}<0.01 ;{ }^{* * *} \mathrm{P}<0.001$ vs. mimic NC+ pcDNA3.1 group; ${ }^{\#} \mathrm{P}<0.001$ vs. miR-524-5p mimic + pcDNA3.1 group. miR, microRNA; NUMB, NUMB endocytic adaptor protein; PCNA, proliferating cell nuclear antigen; NC, negative control; OD, optical density; EV, empty vector.

pressure and proteinuria levels, whereas in normal controls, proteinuria was not detected.

miRNAs serve roles in a number of diseases, such as sclerosis, lung cancer and neurodegenerative diseases like Alzheimer's disease (25). The discovery of dysregulated miRNAs and their gene-regulatory roles in placental development has provided a novel approach for elucidating the underlying mechanisms of pregnancy-specific diseases (26). In the present study, bioinformatics analysis was used to predict the target genes of a specific miRNA, revealing that miR-524-5p was expressed at lower levels in patients with preeclampsia compared with normal controls. miR-524-5p expression in patients with preeclampsia should continue to be monitored in future studies. The predicted target gene of miR-524-5p was NUMB (confirmed by luciferase reporter assay); expression levels of NUMB, a negative regulator of the Notch signaling pathway, were regulated by miR-524-5p (10). Notch is a type of receptor that mediates transmembrane communication and cell fate (27). Bcl-2 is a factor that regulates apoptosis in the intrinsic mitochondrial apoptosis pathway (28). Cyclin D1 is one member of the Cyclin-D family; it is commonly overexpressed and known 
to be a direct target of Jagged1-mediated Notch signaling in breast cancer (29). Inactive CDK6 kinase is reported to disrupt Notch-dependent survival and proliferation by altering the expression levels of Notch target gene (30).

NUMB facilitates the migration and invasion of trophoblastic cells (31). A number of mechanisms have been suggested to explain the dysregulation of NUMB in trophoblastic cells. NUMB serves a key role in asymmetric division (32), as well as in regulating cell recognition, differentiation, tissue renewal and stabilization of the differentiation environment (33). In addition, NUMB acts as an important negative regulator of the Notch signaling pathway (10). The present study demonstrated that miR-524-5p negatively regulated both mRNA and protein levels of NUMB. Viability of HTR-8/SVneo cells was decreased when miR-524-5p expression was inhibited, while cell viability was increased when miR-524-5p was activated using a miR mimic. Subsequently, the effect of miR-524-5p on the proliferative and invasive abilities of HTR-8/SVneo cells were investigated. While low expression levels of miR-524-5p significantly inhibited cell proliferation and migration compared with the control group, activation of miR-524-5p promoted cell proliferation and migration. Next, western blotting was performed to detected the association between miR-524-5p and NUMB, revealing that inhibition of miR-524-5p resulted in upregulation of NUMB. Finally, to investigate the mechanism underlying the miR-524-5p-mediated upregulation of cell proliferation and invasion, Notch signaling pathway-associated proteins were detected via western blotting, including Bcl-2, Notch, cyclin D1 and CDK6. Larger patient cohorts and animal studies are required to validate the results, as these were the two primary limitations of the present research.

In conclusion, the present results demonstrated that miR-524-5p regulated proliferation and invasion of HTR-8/SVneo cells by targeting NUMB to regulate the Notch signaling pathway.

\section{Acknowledgements}

Not applicable.

\section{Funding}

This study was supported by Provincial Natural Science Foundation of China Hainan (grant no. 819MS119).

\section{Availability of data and materials}

The datasets used and/or analyzed during the current study are available from the corresponding author on reasonable request.

\section{Authors' contributions}

LS conceptualized the study and gave final approval of the version to be published. LZ and JS designed and performed the experiments, and wrote and revised the manuscript. LW, RT, XC, DW and HC performed the experiments, analyzed the data and prepared figures and tables. All authors read and approved the final manuscript.

\section{Ethics approval and consent to participate}

The present study was approved by the Ethics Committee of Hainan General Hospital. All patients provided written informed consent prior to participation in the study.

\section{Patient consent for publication}

Not applicable.

\section{Competing interests}

The authors declare that they have no competing interests.

\section{References}

1. Cheng D, Jiang S, Chen J, Li J, Ao L and Zhang Y: Upregulated long noncoding RNA Linc00261 in pre-eclampsia and its effect on trophoblast invasion and migration via regulating miR-558/TIMP4 signaling pathway. J Cell Biochem 120: 13243-13253, 2019.

2. Veerbeek JH, Brouwers L, Koster MP, Koenen SV, van Vliet EO, Nikkels PG, Franx A and van Rijn BB: Spiral artery remodeling and maternal cardiovascular risk: The spiral artery remodeling (SPAR) study. J Hypertens 34: 1570-1577, 2016.

3. Shyu MK, Chen CW, Lin NY, Liao WC, Chen CH, Lin CJ, Huang HC, Lee JJ, Huang MJ, Tseng GF, et al: MUC1 expression is elevated in severe preeclamptic placentas and suppresses trophoblast cell invasion via $\beta 1$-integrin signaling. J Clin Endocrinol Metab 96: 3759-3767, 2011.

4. He J, Xiang D and Yin L: MicroRNA-708 inhibits the proliferation and invasion of osteosarcoma cells by directly targeting ZEB1. Mol Med Rep 19: 3948-3954, 2019.

5. Bhagirath D, Yang TL, Tabatabai ZL, Shahryari V, Majid S, Dahiya R, Tanaka Y and Saini S: Role of a novel race-related tumor suppressor microRNA located in frequently deleted chromosomal locus $8 \mathrm{p} 21$ in prostate cancer progression. Carcinogenesis 40: 633-642, 2019.

6. Chen L, Zhang W, Yan W, Han L, Zhang K, Shi Z, Zhang J, Wang Y, Li Y, Yu S, et al: The putative tumor suppressor miR-524-5p directly targets Jagged-1 and Hes-1 in glioma. Carcinogenesis 33: 2276-2282, 2012.

7. Liu SM, Lu J, Lee HC, Chung FH and Ma N: miR-524-5p suppresses the growth of oncogenic BRAF melanoma by targeting BRAF and ERK2. Oncotarget 5: 9444-9459, 2014.

8. Hromadnikova I, Kotlabova K, Ondrackova M, Pirkova P, Kestlerova A, Novotna V, Hympanova L and Krofta L: Expression profile of C19MC microRNAs in placental tissue in pregnancy-related complications. DNA Cell Biol 34: 437-457, 2015.

9. Yang XR, Sun J, Wang J and Lu YY: Advances in research on cell fate determinant Numb regulating liver cancer. Zhonghua Gan Zang Bing Za Zhi 26: 714-717, 2018 (In Chinese).

10. Zhang J, Shao X, Sun H, Liu K, Ding Z, Chen J, Fang L, Su W, Hong Y, Li H and Li H: NUMB negatively regulates the epithelial-mesenchymal transition of triple-negative breast cancer by antagonizing Notch signaling. Oncotarget 7: 61036-61053, 2016.

11. Zhao C, Guo H, Li J, Myint T, Pittman W, Yang L, Zhong W, Schwartz RJ, Schwarz JJ, Singer HA, et al: Numb family proteins are essential for cardiac morphogenesis and progenitor differentiation. Development 141: 281-295, 2014.

12. Peng H, Wang L, Su Q, Yi K, Du J and Wang Z: MiR-31-5p promotes the cell growth, migration and invasion of colorectal cancer cells by targeting NUMB. Biomed Pharmacother 109: 208-216, 2019.

13. George RM, Biressi S, Beres BJ, Rogers E, Mulia AK, Allen RE, Rawls A, Rando TA and Wilson-Rawls J: Numb-deficient satellite cells have regeneration and proliferation defects. Proc Natl Acad Sci USA 110: 18549-18554, 2013.

14. Rebahi H, Elizabeth Still M, Faouzi Y and Rhassane El Adib A: Risk factors for eclampsia in pregnant women with preeclampsia and positive neurosensory signs. Turk J Obstet Gynecol 15: 227-234, 2018. 
15. ACOG Committee on Obstetric Practice: ACOG practice bulletin. Diagnosis and management of preeclampsia and eclampsia. Number 33, January 2002. American college of obstetricians and gynecologists. Int J Gynaecol Obstet 77: 67-5, 2002.

16. Zhao X, Ren Y, Cui N, Wang X and Cui Y: Identification of key microRNAs and their targets in exosomes of pancreatic cancer using bioinformatics analysis. Medicine (Baltimore) 97: e12632, 2018 .

17. Chen K, Chen H, Zhang K, Sun S, Mo J, Lu J, Qian Z and Yang H: MicroRNA profiling and bioinformatics analyses reveal the potential roles of microRNAs in chordoma. Oncol Lett 14: 5533-5539, 2017.

18. Tan J, Yang L, Liu C and Yan Z: MicroRNA-26a targets MAPK6 to inhibit smooth muscle cell proliferation and vein graft neointimal hyperplasia. Sci Rep 7: 46602, 2017.

19. Song X, Li C, Li J, Liu L, Meng L, Ding H and Long W: The long noncoding RNA uc.294 is upregulated in early-onset pre-eclampsia and inhibits proliferation, invasion of trophoblast cells (HTR-8/SVneo). J Cell Physiol 234: 11001-11008, 2019.

20. Livak KJ and Schmittgen TD: Analysis of relative gene expression data using real-time quantitative PCR and the 2(-Delta Delta C(T)) method. Methods 25: 402-408, 2001.

21. Ishihara N, Matsuo H, Murakoshi H, Laoag-Fernandez JB, Samoto $\mathrm{T}$ and Maruo T: Increased apoptosis in the syncytiotrophoblast in human term placentas complicated by either preeclampsia or intrauterine growth retardation. Am J Obstet Gynecol 186: 158-166, 2002.

22. Iatropoulos MJ and Williams GM: Proliferation markers. Exp Toxicol Pathol 48: 175-181, 1996.

23. Benkő Z, Chaveeva P, de Paco Matallana C, Zingler E, Wright A, Wright D and Nicolaides KH: Validation of competing-risks model in screening for pre-eclampsia in twin pregnancy by maternal factors. Ultrasound Obstet Gynecol 53: 649-654, 2019.

24. Haram K, Bjørge L and Guttu K: Pathophysiology and clinical manifestations in pre-eclampsia. Tidsskr Nor Laegeforen 120: 1426-1431, 2000 (In Norwegian).

25. Hewel C, Kaiser J, Wierczeiko A, Linke J, Reinhardt C, Endres K and Gerber S: Common miRNA patterns of Alzheimer's disease and parkinson's disease and their putative impact on commensal gut microbiota. Front Neurosci 13: 113, 2019.
26. Liang W, Song WY, Xie Y, Hu LL, Hou XM, Wang R, Gao Y, Zhang JN, Zhang L, Li WW, et al: miR-181a-5p suppresses invasion and migration of HTR-8/SVneo cells by directly targeting IGF2BP2. Cell Death Dis 9: 16, 2018.

27. Qi S, Lei Y,Zhao L, Mu YL, Li M,Zhao X, Chen ZJ and Zhang H: Melatonin inhibits $17 \beta$-estradiol-induced migration, invasion and epithelial-mesenchymal transition in normal and endometriotic endometrial epithelial cells. Reprod Biol Endocrinol 16: 62, 2018.

28. Frenzel A, Grespi F, Chmelewskij W and Villunger A: Bcl2 family proteins in carcinogenesis and the treatment of cancer. Apoptosis 14: 584-596, 2009.

29. Reedijk M, Reedijk M, Reedijk M, Cohen B, Shimizu M, Ng N, Bukhman Y, Pan J and Dering J: Cyclin D1 is a direct target of JAG-mediated Notch signaling in breast cancer. Cancer Res 69 (Suppl 24): S2150, 2009.

30. Hu MG, Deshpande A, Schlichting N, Hinds EA, Mao C, Dose M, Hu GF, Van Etten RA, Gounari F and Hinds PW: CDK6 kinase activity is required for thymocyte development. Blood 117: 6120-6131, 2011.

31. Wang J, Zhou T, Sun Z, Ye T, Zhou S, Li J, Liu Y, Kong L, Tang J, Liu D and Xing HR: Zeb1 regulates the symmetric division of mouse lewis lung carcinoma stem cells through Numb mediated by miR-31. Int J Biol Sci 14: 1399-1410, 2018.

32. Wu YC, Lee KS, Song Y, Gehrke S and Lu B: The bantam microRNA acts through Numb to exert cell growth control and feedback regulation of Notch in tumor-forming stem cells in the Drosophila brain. PLoS Genet 13: e1006785, 2017.

33. Tosoni D, Zecchini S, Coazzoli M, Colaluca I, Mazzarol G, Rubio A, Caccia M, Villa E, Zilian O, Di Fiore PP and Pece S: The Numb/p53 circuitry couples replicative self-renewal and tumor suppression in mammary epithelial cells. J Cell Biol 211: 845-862, 2015.

This work is licensed under a Creative Commons Attribution-NonCommercial-NoDerivatives 4.0 International (CC BY-NC-ND 4.0) License. 